\title{
Assemblage of encrusting organisms on floating marine debris along the west coast of Qatar
}

Faculty and PostDoc,

Science and Engineering
Jassim A. Al-Khayat, S. Veerasingam, V.M. Aboobacker, P. Vethamony

UNESCO Chair in Marine Sciences, Environmental Science Center, Qatar University, P.O. Box 2713, Doha, Qatar

ABSTRACT: The floating marine debris (FMD) and the associated rafting communities are one of the major stressors to ecosystem services, global biodiversity and economy and human health. In this study, assemblages of encrusting organisms on different types of stranded FMD along the west coast of Qatar, Arabian Gulf (hereafter referred to as 'Gulf') were examined. The analysis showed 18 fouling species belonging to 5 phyla (Annelida, Anthropoda, Bryozoa, Mollusca and Porifera) on the FMD. The most abundant fouling species were the encrusting Amphibalanus amphitrite, polychaete Spirobranchus kraussii, Bryozoan species and Megabalanus coccopoma. More number of taxa were found on larger size FMD than on smaller FMD. Some of the barnacle rafting types were found to be non-indigenous species. The central and northwest parts of the Qatar had more FMD and fouled species than in other locations. The present stud confirmed that huge amount of bio-fouled FMD items, causing great damage to biodiversity, drift in the surface layer of ocean under the influence of coastal dynamics, and eventually strand onto the beaches. We propose a simple, but an effective management plan for FMD and associated organisms at regional scale to restore the biodiversity, sustainability and health of the marine ecosystem in the Gulf.

\section{INTRODUCTION}

An explosive increase of Floating marine debris (FMD) and associated assemblage of marine invertebrates in the oceans may act as a possible dispersal vehicle for spreading invasive species, which is one of the greatest stressors to global biodiversity. FMD acts as a vector for both short and long-distance transport of invasive species. Plastic FMD item pose extensive ecological stressors including alteration of pelagic habitats and transport of alien species. Introduction of several invasive species a hitchhikers is considered as a pest on farmed molluscs, which harms the native species and marine ecosystem. In most of the major oceanic regions, nearly 400 taxa have been found as rafting species on FMD Therefore, understanding the biosecurity implications associated witl rafting species is a crucial step to identify, monitor and mitigate its impacts. In the last two decades, many research studies have been carried out to assess the risk of invasive species through FMD in the world's oceans and marginal seas. Apparently, only one study has illustrated the distribution of invasive species associated with FMD in the Gulf along the Iran coast. Therefore, in this study the distribution and transportation of benthic invertebrates encrusting on FMD along the west coast of Qatar have been studied

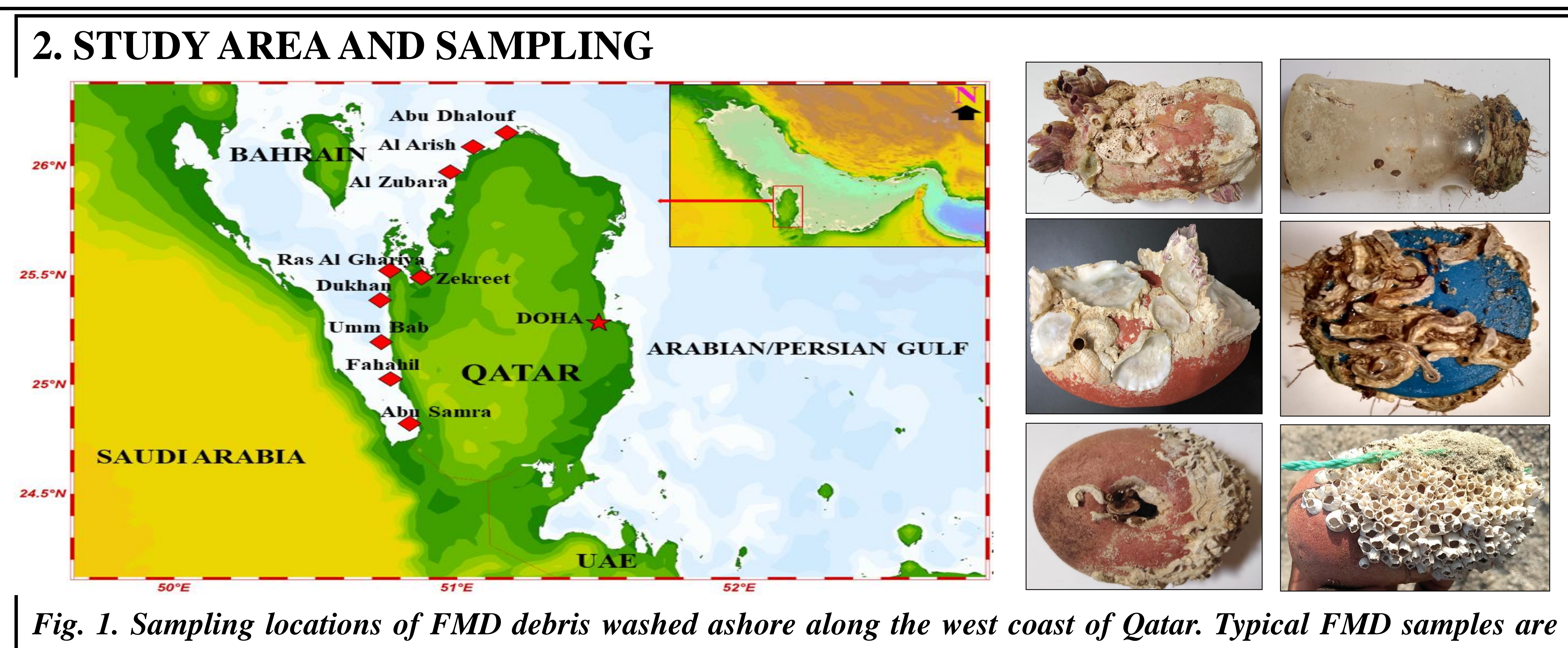

\section{Fig. 1. Sampth}

We conducted FMD surveys at 9 beaches along the west coast of Qatar in July 2020 (Fig.1) At each beach, sampling was conducted for nearly one hour at daylight in the intertidal and berm line. Debris items including plastics (mostly plastic bottles, fishing buoys and Styrofoam), wood, metal cans and glass bottles were sampled. Only the fouled debris items were considered for the present study. The collected FMD samples were packed in aluminum foil bags. Buoyancy assays with all collected debris displayed that nearly all of them were positively buoyant, and these FMD must have reached the shoreline along with currents

\section{ANALYTICAL METHODS}

The collected FMD samples were cleaned and photographed. FMD items with three-dimensional shapes (e.g., PET bottles, metal cans and fishing buoys) were photographed at both sides (front and back side of FMD), and created two distinct images (Shahdadi et al. ${ }^{[2]}$ ). The data of encrusting organisms on FMD from these twe photographs were then pooled. Later, macroscopic specimens of all FMD were examined using a dissecting microscope, and identified using available literature ${ }^{2,3,4}$ Based on the prevalence of FMD items collected along the west coast of Qatar, the coverage of encrusting species was calculated. Coral Point Count with Excel extension (CPCe) version 4.1 was used to calculate the total surface area of FMD items and each species on the photographs. The cluster analysis (CA) was carried out using the Minitab 19 statistical software package. CA was conducted to classify both the sampling locations and the abundance of rating species.

\section{RESULTS AND DISCUSSIOI}

In this study, a total of 70 FMD items were found on the surveyed beaches. A total of 18 encrusting species belonging to 5 phyla were identified (Table 1 ). The mos abundant fouling phyla were arthropoda $(588 ; 65.3 \%)$, annelida $(190 ; 21.1 \%)$, mollusca $(92 ; 10.2 \%)$, bryozoa (18; 2\%) and porifera (12; 1.3\%) (Fig. 2). The first number in the parentheses is the phyla number. The overall composition of rafting species is as follows: barnacle Amphibalanus amphitrite (54.99 \%), polychaete Spirobranchus kraussii (18.09 \%), Bryozoan sp. (10.97\%) and Megabalanus coccopoma (4.13\%). The barnacle Amphibalanus amphitrite was the most abundant rafting species found in the central and northwest parts of Qatar. In some cases, anthropoda (Megabalanus coccopoma) was still alive. However, in most locations, A. amphitrite was found dead very recently. It could be that the FMDs might have been deposited on the shore and exposed to air and sunlight for a longer time. The highest coverage of rafting species was observed at Al Arish (24.8\%) and the lowest at Fahahil (3.1\%) (Fig. 3a). The coverage of rafting species was the highest on plastics (85.26 \%), whereas it was the lowest on metals $(0.79 \%)$. Cluster analysis results classified both the sampling locations and the abundance of rating species (Fig. 4a, b). Our results confirm that there is a variety of benthic organisms attached to the transboundary plastics and fishing related items deposited along the west coast of Qatar. In most of the sampling beaches, $A$. amphitrite was found as the dominant rafting species.
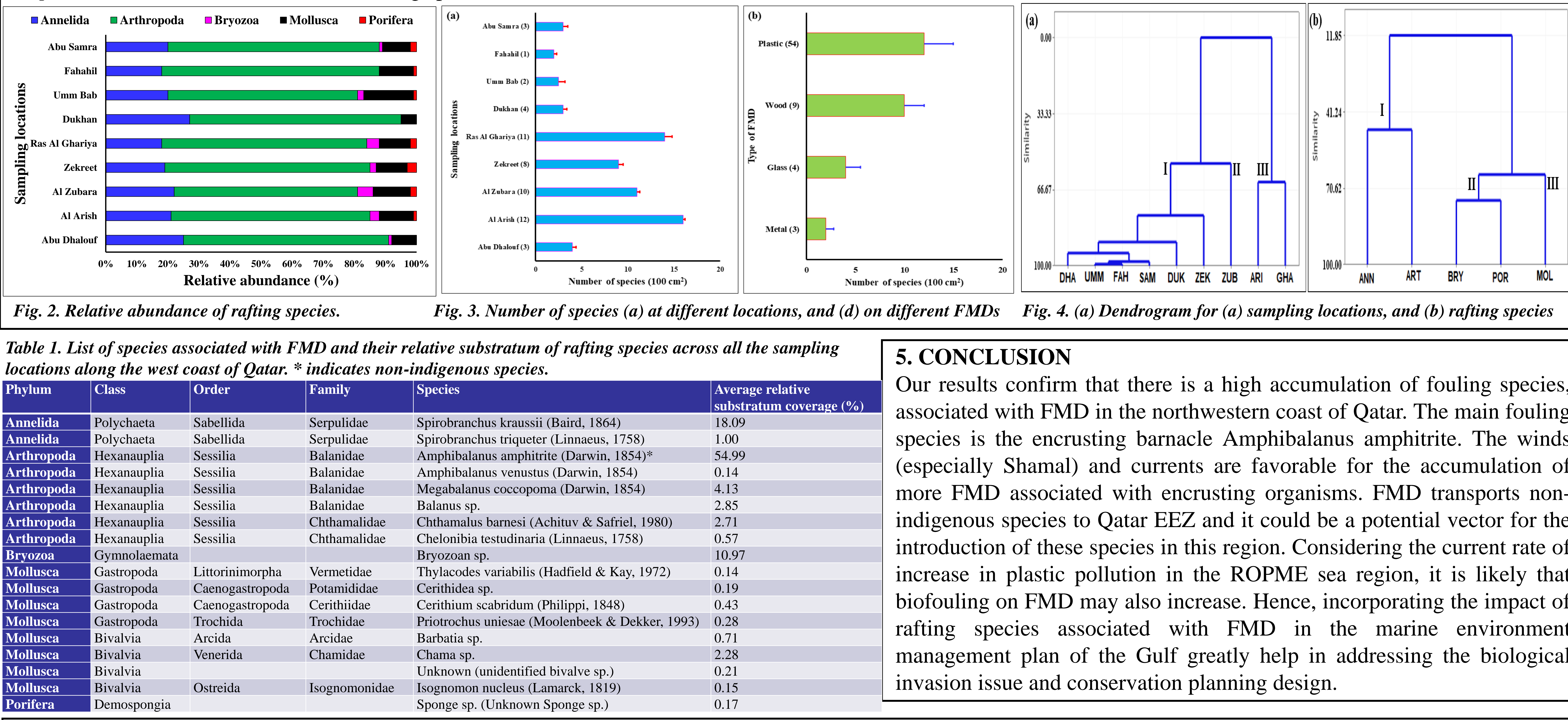

\begin{abstract}
5. CONCLUSION
Our results confirm
Our results confirm that there is a high accumulation of fouling species, associated with FMD in the northwestern coast of Qatar. The main fouling species is the encrusting barnacle Amphibalanus amphitrite. The winds (especially Shamal) and currents are favorable for the accumulation of more FMD associated with encrusting organisms. FMD transports nonindigenous species to Qatar EEZ and it could be a potential vector for the introduction of these species in this region. Considering the current rate of increase in plastic pollution in the ROPME sea region, it is likely that biofouling on FMD may also increase. Hence, incorporating the impact of rafting species associated with FMD in the marine environment management plan of the Gulf greatly help in addressing the biological invasion issue and conservation planning design.
\end{abstract}

6. ACKNOWLEDGEMENT: This work is funded by the International Research Collaboration Co-funding program of Qatar University (IRCC-2019-002). Part of the objectives of UNESCO Chair in Marine Sciences, namely, Marine Pollution, was implemented through this project 